\section{(2)} OPEN ACCESS

\title{
Multiprofessional intervention to improve adherence to medication in stroke patients: a study protocol for a randomised controlled trial (ADMED AVC study)
}

\author{
Sophie Khettar (1) 1,2 Sophie Jacquin Courtois, ${ }^{3,4}$ Jacques Luaute, ${ }^{3,4}$ Evelyne Decullier, ${ }^{5}$ \\ Sylvie Bin, ${ }^{5}$ Marine Dupuis, ${ }^{5}$ Laurent Derex, ${ }^{6,7}$ Laura Mechtouff, 7,8 \\ Norbert Nighoghossian, ${ }^{7,9}$ Claude Dussart, ${ }^{2}$ Gilles Rode, ${ }^{3,4}$ Audrey Janoly-Dumenil ${ }^{1,2}$
}

\begin{abstract}
- Additional material is published online only. To view please visit the journal online (http://dx.doi.org/ 10.1136/ejhpharm-2020002425).
\end{abstract}

For numbered affiliations see end of article.

\section{Correspondence to} Dr Sophie Khettar, EA 4129 P2S Parcours Santé Systémique, Faculté de Médecine Laënnec, 7-11 rue Guillaume Paradin 69372 Lyon cedex 08, France; sophie. khettar@chu-lyon.fr

Received 20 June 2020 Revised 17 July 2020 Accepted 21 July 2020
D) Check for updates

(C) European Association of Hospital Pharmacists 2020. Re-use permitted under CC BY-NC. No commercial re-use. Published by BMJ.

To cite: Khettar S, Jacquin
Courtois S, Luaute J, et al.
Eur J Hosp Pharm Epub
ahead of print: [please
include Day Month Year].
doi:10.1136/
ejhpharm-2020-002425

\section{ABSTRACT}

Introduction Adherence to secondary preventive medications is often suboptimal in patients with stroke, exposing them to an increased risk of recurrent cerebral and/or cardiovascular events. Effective actions in the long term to improve adherence to medication are needed. The study will evaluate the efficacy of a collaborative multiprofessional patient-centred intervention conducted by a pharmacist on adherence to secondary preventive medication in stroke survivors.

Methods and analysis This is a multicentre clusterrandomised controlled trial. Two groups of 91 patients (intervention vs standard care) will be recruited. The clinical pharmacist intervention targeting secondary preventive medication will consist of three parts over 1 year: (1) an individual semi-structured interview at hospital discharge; (2) follow-up telephone interviews at 3, 6 and 9 months after discharge; and (3) a final individual semi-structured interview 1 year after discharge. Information on patient follow-up will be shared with the general practitioner and the community pharmacist by sending a report of each interview. The primary outcome is adherence to medication during the 12 months after hospital discharge, assessed using a composite endpoint: the medication possession ratio associated with a self-administered questionnaire.

Ethics and dissemination The local ethics committee, the national committee for use of personal data in medical research and the national data protection agency approved the study. The sponsor has no role in study design; collection, analysis and interpretation of data; or report writing.

Discussion This pharmacist-led educational programme has the potential to significantly improve adherence to medication in stroke survivors which could lead to a decrease in recurrent cerebral and/or cardiovascular events.

Trial registration number NCT02611440

\section{INTRODUCTION}

After a stroke the effectiveness of secondary prevention to prevent the recurrence of vascular events requires optimum adherence by the patient. ${ }^{1-7}$ Unfortunately, suboptimal adherence to medication is often observed, with rates ranging from $<10 \%$ to $>95 \%$ according to the methodology used, the time of measurement and the drug concerned. ${ }^{8-10}$

Among published studies investigating programmes aimed at improving adherence to post-stroke medication, several differences are observed. For instance, articles reporting randomised controlled trials (RCTs) of interventions that significantly improved adherence to medication among stroke survivors ${ }^{11-13}$ investigated several types of educational programmes that took place in different locations (at home or hospital) and at various intervals after stroke. Furthermore, adherence was measured using several different methods, which makes it difficult to interpret the evidence. They also did not specify the profession of the person who interacted with the patient. It is therefore currently unclear how to improve medication adherence after a stroke.

It is noteworthy that the role of pharmacists in such programmes has not been extensively investigated, unlike that of nurses. ${ }^{14-20}$ However, none of the RCTs that tested nurse-led interventions reported a significant improvement in adherence to secondary preventive medication after an ischaemic stroke. ${ }^{1415171920}$ In the few studies that were pharmacist-led, the results were positive but no conclusion could be made as to the efficacy of such interventions. ${ }^{21-23}$ The study by Hedegaard et $a l^{22}$ did not report a significant improvement in adherence to secondary preventive medication, persistence or clinical outcomes in patients receiving a pharmacist-led intervention, possibly due to several limitations such as a carryover between the intervention and control groups or the establishment of a secondary prevention clinic during the study, which may explain the unexpected high adherence rates in both groups. Lummis et $a l^{21}$ described activities carried out by the pharmacist in the healthcare team in a stroke clinic but unfortunately this study was not a RCT, which limits the conclusions. McAlister et $a l^{23}$ reported significantly better achievement of systolic blood pressure and low-density lipoprotein targets among stroke patients addressed by pharmacist-led care compared with nurse-led care, but no difference regarding adherence to medication was found. Furthermore, pharmacist interventions included initiation and titration of medication according to Canadian guidelines that are not reproducible in France.

A pharmacist-led intervention still has the potential to be effective because pharmacist interventions have been reported to have a significant impact on adherence to medication for general cardiovascular prevention $^{24-26}$ and in reducing cardiovascular 
disease risk factors. ${ }^{27-31}$ Furthermore, therapeutic education conducted by a pharmacist with stroke survivors was also found to improve patient quality of life. ${ }^{32}$ A recent description of a pharmacist-led stroke prevention clinic seems to show the relevance of interventions (medication reconciliation, updating medication list, education on stroke risk reduction and reinforcement of medication adherence on a regular basis) on several controls of risk factors; however, this was not formally evaluated by a RCT. ${ }^{33}$

Further studies are needed to explore the potential of pharmacist-led interventions to improve adherence to medication in patients with stroke. As a drug expert, the pharmacist can act on numerous factors that have a role to play in adherence to medication by patients with chronic diseases.

In this context, the ADMED-AVC study was selected as part of a call for projects put in place by the French Ministry of Health (Direction Générale de l'Offre de Soins, DGOS). The funding obtained from the French national healthcare system research programme on the performance of healthcare systems (Programme de Recherche sur la Performance du Système des Soins, PREPS; no 13-0533) made it possible to carry out a prospective multicentre RCT to evaluate the efficacy of a collaborative multiprofessional patient-centred intervention conducted by a clinical pharmacist on adherence to secondary preventive medication in stroke survivors. The study protocol is reported here.

\section{METHODS AND ANALYSIS \\ Study design}

This is an open-label multicentre RCT comparing a pharmacistdriven intervention with standard care. Inpatients will be recruited in the stroke unit $(\mathrm{n}=1)$ or the neurological rehabilitation unit $(n=5)$ of French university hospitals.

The level of randomisation will be the centres and not the patient. Each centre will be assigned to one of the two periods (intervention vs standard care): for example, to either intervention followed by standard care, or standard care followed by intervention, each with a 1-month washout between the two periods. Each centre represents a cluster and will participate in both strategies, therefore each group will comprise the six centres. Eligible patients will be offered to participate in the study according to the assigned period of the centre at the time of their discharge. The computer-generated randomisation will be conducted before the start of the study by the clinical research methodology unit of the Lyon teaching hospitals (Hospices Civils de Lyon, Lyon, France) using SAS software V9.4 (Cary, North Carolina, USA). The follow-up period for the study will be 12 months. A flowchart of the trial design is shown in figure 1 .

\section{Patient recruitment}

Inpatients (post-stroke hospitalisation) with ischaemic stroke will be included if they are aged 18 years or more, taking medication including an antiplatelet or anticoagulant agent with at least an antihypertensive agent and/or a statin, are returning home at hospital discharge, have no cognitive or major psychiatric disorder and have sufficient autonomy for the management of medication at home (Barthel index >30). The study will be proposed to inpatients by the investigator in tandem with the pharmacist once hospital discharge is planned. The investigator will present the study and sign the consent with the patient, and the pharmacist will then take charge of the pharmaceutical evaluation.

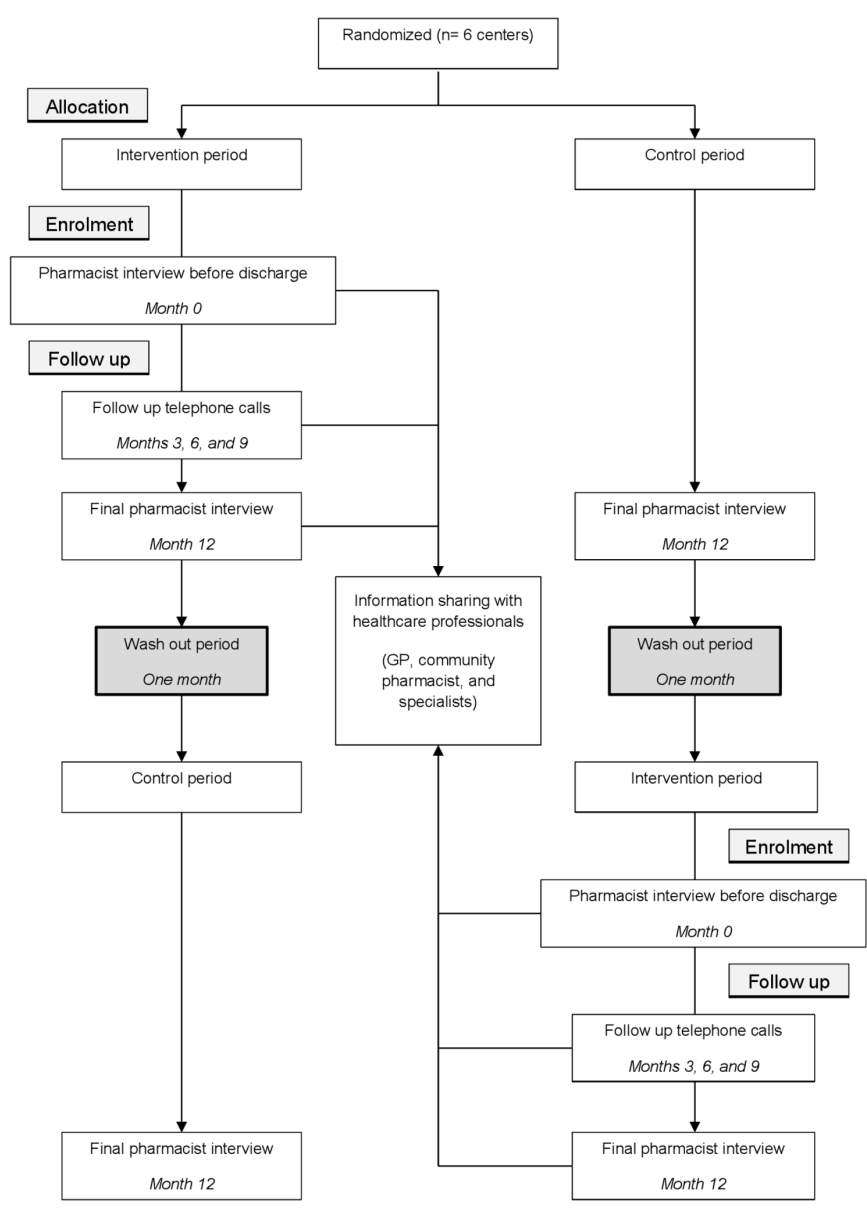

Figure 1 Flowchart of trial design. GP, general practitioner.

Inpatients will not be included if they do not have a usual community pharmacy (or more than two usual community pharmacies). Indeed, the ADMED-AVC project seeks to create the link between healthcare professionals around the stroke patient, which appears to be difficult if there are too many participants and also makes it difficult to retrieve refill data.

\section{Study groups}

The study comprises two groups, the standard care group and the intervention group.

\section{Standard care group}

Aside from medication review which is carried out routinely during hospitalisation, patients in the standard care group will receive no specific intervention by the clinical pharmacist. A pharmacist will give a follow-up diary to the patients before hospital discharge and will set up a single interview with them to be conducted at the hospital 1 year after discharge. During this interview every drug-related problem reported in the patient follow-up diary will be discussed. The primary outcome will be measured at this time.

\section{Intervention group}

The intervention will be driven by the clinical pharmacist and will consist of (1) an individual semi-structured interview with the patient at hospital discharge (day 0); (2) three follow-up telephone interviews at 3,6 and 9 months with the patient to reinforce adherence to medication; and (3) an individual interview conducted at the hospital 12 months after discharge. Each 
interview between the clinical pharmacist and patient (at hospital and telephone calls) will be formally summarised by the pharmacist. This report will be sent to the patient's general practitioner (GP), specialists when appropriate, and his/her usual community pharmacist, with the indication of any prescription changes and drug-related problems (eg, interactions or adverse effects). This report will be co-signed by the investigator and the pharmacist. Healthcare professionals involved in the patient's long-term management will thus have the same level of information about the patient for optimised follow-up.

\section{Patient interviews}

Interview before discharge

A trained hospital clinical pharmacist will conduct a semistructured interview with the patient lasting approximately $60 \mathrm{~min}$. The aim is to inform them about the disease, the role of secondary preventive medication and the significance of lifestyle and dietary recommendations. Above all, the clinical pharmacist will check the patient's discharge prescription and apply a medication reconciliation process before the interview.

The first part of the interview will explore patient barriers to medication adherence to understand and adapt her/his presentation to the patient. The pharmacist will then explain changes to treatment before focusing on secondary stroke prevention medication with tailored information. The objective will be to explain the treatments and to answer questions on the dayto-day management of treatments. The pharmacist will deal with practical situations to assess patients' self-management skillsfor instance, situations with an increased iatrogenic risk such as bleeding with an anticoagulant agent or muscle pain with a lipidlowering agent.

At the end of the interview the pharmacist will draw up a discharge plan, ${ }^{33}$ which will consist of a review of discharge medication and reconciliation with the patient's drug regimen at admission, and a medication plan in collaboration with the patient. The pharmacist will synthesise all important information and prepare a report of the discharge medication, indicating any prescription changes to the patient's GP/specialists and the community pharmacist.

Participants will also receive educational brochures to support oral information. The educational material about stroke preventive treatments (explanatory document for each pharmacological class) were developed by pharmacists and a physical and rehabilitation medicine (PRM) physician, validated by physicians and patients from a stroke association, endorsed by the Hospices Civils de Lyon and the French physical and rehabilitation medicine society (Société Française de Médecine Physique et Réadaptation, SOFMER). A guide developed by the French Ministry of Health for implementation of lifestyle and dietary recommendations will also be given. Finally, the patient will receive a follow-up diary to report potential treatment-related difficulties, treatment modifications, side effects and hospitalisations.

\section{Telephone interviews}

The patient will be contacted by telephone at 3, 6 and 9 months after discharge. The aim of these telephone interviews is to review treatment modifications, drug-related problems and to reinforce adherence to medication. In particular, drug-related problems such as side effects, drug interactions and problems related to medication delivery will be discussed with the patient. The pharmacist will remind the patient and insist on the control of risk factors by following lifestyle and dietary recommendations, adherence to medication and understanding the long-term benefit of secondary preventive medication. These discussions will allow changes implemented by the patient in her/his life following her/his stroke, both in terms of medication and lifestyle, to be addressed, or to help in their implementation if necessary.

\section{Interview at 12 months}

A final individual interview 1 year after discharge will be conducted by the clinical pharmacist at the hospital. The patient will first be given self-administered questionnaires (see below), including a medication adherence questionnaire (8-item questionnaire). After this the last follow-up interview will be conducted to review treatment modifications and drug-related problems (side effects, drug interactions and problems related to medication delivery).

The study procedures and assessments are outlined in the Standard Protocol Items: Recommendation for Interventional Trials (SPIRIT) checklist and SPIRIT (table 1).

\section{Primary outcome}

The primary outcome will be adherence to secondary preventive medication measured at 1 year after hospital discharge. A patient will be considered adherent if (1) the medication possession ratio for each secondary preventive drug (defined as number of days of medication available to the patient divided by total number of days with prescription in the period of the study) is greater than $80 \%{ }^{34}$; and (2) the patient has an adherence score greater than $6 / 8$ for the entire secondary preventive treatment as assessed using a self-administered adherence questionnaire. For instance, if the medication possession ratio for the antihypertensive agent and the statin are greater than $80 \%$ but is $60 \%$ for the antiplatelet agent, the patient is categorised as non-adherent even if the self-reported adherence score for the overall treatment is greater than $6 / 8$. The community pharmacist will be contacted by telephone by the hospital pharmacist to assess the rate of refill for each medication.

At 12 months, at least one of the following drugs will need to still be prescribed for evaluation: antiplatelet, oral anticoagulant, angiotensin converting enzyme inhibitor, calcium inhibitor, diuretic, statin (pharmacological classes selected according to current French guidelines). ${ }^{1}$

\section{Secondary outcomes}

Secondary outcomes, which include adherence to each secondary preventive drug, quality of life as assessed by the Short Form 36 (SF-36) Health Survey questionnaire, ${ }^{35}$ belief/representation about the disease as assessed by the Brief Illness Perception Questionnaire (Brief IPQR $)^{36}$ and patient satisfaction regarding treatment as assessed by the Treatment Satisfaction with Medicines Questionnaire (SATMED-Q) ${ }^{37}$ are shown in table 1. Patient knowledge about the disease and treatments, and also lifestyle (eg, diet, exercise) will be assessed by self-administered questionnaires developed by pharmacists according to French guidelines. ${ }^{38}$ Patient satisfaction about the intervention programme and his/her relationship with the healthcare professionals will be collected through a Likert scale questionnaire, as will the community pharmacist's and GP's satisfaction. The patient selfadministered questionnaires will be provided by the hospital pharmacist and will be completed just before the interview (months 0 and 12). The questionnaires for the community pharmacist and the GP will be completed by telephone or will be sent and returned by mail. 
Table 1 Standard Protocol Items: Recommendation for Interventional Trials (SPIRIT)

\begin{tabular}{|c|c|c|c|c|c|c|}
\hline \multirow{3}{*}{ TIME POINT } & \multicolumn{6}{|l|}{ Study period } \\
\hline & \multirow{2}{*}{$\begin{array}{l}\text { Hospital stay } \\
-1 \text { to }-7 \text { days }\end{array}$} & \multirow{2}{*}{$\begin{array}{l}\text { Hospital discharge } \\
0\end{array}$} & \multicolumn{3}{|l|}{ Follow-up } & \multirow{2}{*}{$\begin{array}{l}\text { Close-out } \\
\text { M12 }\end{array}$} \\
\hline & & & M3 & M6 & M9 & \\
\hline \multicolumn{7}{|l|}{ ENROLMENT } \\
\hline Eligibility screen & $\mathrm{X}$ & & & & & \\
\hline Informed consent & $\mathrm{X}$ & & & & & \\
\hline \multicolumn{7}{|l|}{ INTERVENTIONS } \\
\hline Medication reconciliation & & $X^{*}$ & & & & \\
\hline Medication review & & $\mathrm{X}$ & & & & \\
\hline Interview before discharge & & $X^{*}$ & & & & \\
\hline $\begin{array}{l}\text { Providing educational brochures, discharge plan, } \\
\text { medication plan }\end{array}$ & & $X^{*}$ & & & & \\
\hline Providing follow-up diary & & $x$ & & & & \\
\hline Information sharing with healthcare professionals & & $X^{*}$ & $X^{*}$ & $X^{*}$ & $X^{*}$ & $X^{*}$ \\
\hline Telephone interviews & & & $X^{*}$ & $X^{*}$ & $X^{*}$ & \\
\hline Final interview & & & & & & $\mathrm{X}$ \\
\hline \multicolumn{7}{|l|}{ ASSESSMENTS } \\
\hline History, demographics & & $\mathrm{x}$ & & & & \\
\hline \multicolumn{7}{|l|}{ PRIMARY OUTCOME } \\
\hline Medication possession ratio & & & $X^{*}$ & $X^{*}$ & $X^{*}$ & $X$ \\
\hline Adherence scale & & $\mathrm{x}$ & & & & $\mathrm{x}$ \\
\hline \multicolumn{7}{|l|}{ SECONDARY OUTCOMES } \\
\hline Blood glucose control & & $X$ & & & & $X$ \\
\hline Lipid control & & $x$ & & & & $x$ \\
\hline Abdominal girth & & $X$ & & & & $X$ \\
\hline Blood pressure & & $x$ & & & & $x$ \\
\hline Quality of life (SF-36) & & $X$ & & & & $\mathrm{X}$ \\
\hline Belief/representation about the disease (Brief IPQR) & & $X$ & & & & $X$ \\
\hline Knowledge about the disease and the treatments & & $\mathrm{X}$ & & & & $\mathrm{X}$ \\
\hline Lifestyle & & $X$ & & & & $x$ \\
\hline Number, type and severity of iatrogenic events & & & & & & $X$ \\
\hline $\begin{array}{l}\text { Proportion of patients having recurrent stroke or other } \\
\text { major adverse cardiovascular event }\end{array}$ & & & & & & $\mathrm{x}$ \\
\hline Proportion of patients readmitted to hospital & & & & & & $\mathrm{X}$ \\
\hline Adherence to each secondary preventive drug & & & & & & $X$ \\
\hline Patient satisfaction regarding treatments (SATMED-Q) & & & & & & $X$ \\
\hline Patient satisfaction regarding the intervention programme & & & & & & $X^{*}$ \\
\hline $\begin{array}{l}\text { Community pharmacist's satisfaction regarding the } \\
\text { intervention programme }\end{array}$ & & & & & & $X^{*}$ \\
\hline $\begin{array}{l}\text { General practitioner's satisfaction regarding the } \\
\text { intervention programme }\end{array}$ & & & & & & $X^{*}$ \\
\hline
\end{tabular}

\section{Statistical analysis}

We hypothesised that the proportion of adherent patients would reach $80 \%$ in the control group and $95 \%$ in the intervention group. To achieve $80 \%$ power with a two-sided alpha level of $0.05,152$ patients are needed (76 in each group). To take into account loss to follow-up, a total sample size of 182 patients will be included (91 in each group). This sample size is global and there is no target number of patients by cluster.

The data will be analysed using SAS software V9.4 (Cary, North Carolina, USA). Two populations will be defined: the intention-to-treat population will include all included patients and the per protocol population will include only patients without major deviation from the protocol.

Patient characteristics will be reported using descriptive statistics (number, mean, SD, median and range for quantitative variables, and number and percent for categorical variables) for each group at global and cluster level.

The main outcome analysis will be conducted on the intentionto-treat population (main analysis); patients with missing information at 1 year (due to death, loss to follow-up and withdrawal of consent) will be considered non-adherent in the analyses. The effect of the intervention on adherence to medication will be quantified and tested using a logistic regression adjusted on cluster and period. The same analysis will be conducted on the per protocol population.

For secondary outcome analyses, differences in proportions between groups (standard care vs intervention) will be compared using logistic regression adjusted for cluster and period. For quantitative outcome, an analysis of variance (ANOVA) will be used to compare mean values between the two groups adjusted

Khettar S, et al. Eur J Hosp Pharm 2020;0:1-7. doi:10.1136/ejhpharm-2020-002425 
for cluster and period. The mean scores of each questionnaire will be compared between the two groups at 1 year. For the SF36 and Brief IPQR questionnaires, the difference between the mean scores at baseline and at 1 year will also be calculated and compared between the two groups. We will analyse the Brief IPQR mean score at baseline to compare adherent and nonadherent patients. The mean number of side effects and readmissions will be compared between the two groups, and we will also compare mean values of collected laboratory data (fasting glucose, glycated haemoglobin, triglycerides, HDL cholesterol, LDL cholesterol and total cholesterol levels) at 1 year.

For each test a two-sided $\mathrm{p}$ value of $<0.05$ will be considered significant.

\section{ETHICS AND DISSEMINATION}

\section{Ethics approval and informed consent}

The local ethics committee (Comité de protection des personnes Sud Est II) approved the study for all centres (online supplemental file). The national committee for use of personal data in medical research (Comité Consultatif sur le Traitement de l'Information en matière de Recherche dans le domaine de la Santé, CCTIRS) and the national data protection agency (Commission nationale de l'informatique et des libertés, CNIL) approved the study. Prior written informed consent of recruited patients will be obtained. This trial was registered with ClinicalTrials.gov (number NCT02611440).

\section{Monitoring}

The promoter will delegate a clinical research associate to monitor the study in accordance with the existing regulations. Newsletters will be sent to those involved in the research to provide information about activities relating to the clinical trial such as an overview of study progress and information about upcoming changes. According to the French law, the following will be reported to the national medicines agency (Agence Nationale de Sécurité du Médicament et des produits de santé, ANSM) and to the local ethics committee: every unexpected serious adverse effect, any new safety information and annual safety reports. No adverse event related to study participation is expected, so no independent monitoring committee will be needed.

\section{Data management}

Only those involved and identified in the research will have access to the data entry software EOL via Medsharing. The data entry in the electronic case report form (e-CRF) will be controlled for value ranges and outliers. The data will be hosted in a clean room by the company CLARANET. Access to this application will be secure and via https://www.essaionline.com/default.asp using a personal login and password. The data stored on Medsharing's servers will be duplicated in real-time on multiple disks with robotic backup hourly, daily, weekly and monthly. Data protection will be guaranteed during the exchanges between the user and the servers. The information flowing between the client and the server will be encrypted using a strong encryption technique. Access will be secured by a firewall and only network administrators and authorised persons from the clinical research methodology unit will access the directory.

\section{Confidentiality}

In accordance with the provisions concerning the confidentiality of data available to persons in charge of quality control of research involving humans (Article L.1121-3 of the Public Health
Code), in accordance with the provisions relating to the confidentiality of information in particular clinical trials, the persons who lend themselves to them and the results obtained (article R. 5121-13 of the Public Health Code), the persons having direct access to the data will take all the precautions necessary to ensure the confidentiality of the information relating to the trials, the persons who are suitable for them and, in particular, with regard to their identity and the results obtained. These persons, as well as the investigators themselves, will be subject to professional secrecy (according to the conditions defined by articles 226-13 and 226-14 of the Penal Code).

During and after the research involving the human persons, the data collected on the individuals who will be amenable to it and transmitted to the sponsor by the investigators (or any other authorised person) will be made anonymous. Only the first letter of the subject's name and the first letter of his/her first name will be recorded, accompanied by a coded number specific to the study indicating the order of inclusion of the subjects.

\section{Dissemination}

The investigators and the project manager will be responsible for the publications reporting the results of the study. The sponsor has no role in study design; collection, analysis and interpretation of data; or report writing.

\section{DISCUSSION}

\section{Clinical pharmacist-driven intervention}

The clinical pharmacist intervention relying on the patientcentred approach was designed to cover both intentional and non-intentional poor adherence. ${ }^{7}$ By providing patient-tailored detailed information about treatments, the pharmacist intervention will attempt to modify erroneous beliefs of patients with stroke about medication and intentional non-adherence. In parallel, a precise medication plan (when, where and how to take medications), provision of educational brochures and dealing with practical situations to assess patients' self-management skills will be expected to improve non-intentional non-adherence.

The design of the pharmacist-led intervention targets many barriers negatively influencing adherence to stroke medication: difficulties in taking medication, ${ }^{39}$ lack of understanding of information provided by health professionals, ${ }^{40}$ lack of follow-up by health professionals, ${ }^{4041}$ erroneous beliefs of benefits of treatments, ${ }^{40} 42-44$ lack of knowledge about the disease and treatments ${ }^{1039} 4043$ and concerns about adverse effects. ${ }^{404143}$

It has been highlighted in a qualitative French study exploring patient perceptions of stroke and secondary preventive treatments that patients have difficulties in understanding the severity of their condition when they are asymptomatic as well as the objectives of post-stroke medication; stroke survivors expressed a need for more information on stroke and its treatments (dosage, schedule, benefits/side effects). ${ }^{39}$ The present study embraces all these aspects with an intervention that assesses barriers to medication adherence and aims to improve patient knowledge about stroke as well as stroke medication by providing tailored information.

Other strengths of the study include the timing of the interventions, as the pharmacist intervention will be initiated at a pertinent moment (before discharge from the stroke unit or from the neurological rehabilitation unit for those with disabilities) and will continue after hospital discharge. In this way, all major information and explanations about treatments will be given and summarised to the patient just before discharge, and will be delivered on a regular basis for 1 year to reinforce adherence. 
More generally, the intervention includes various approaches that have demonstrated efficacy in improving adherence to medication (such as dealing with barriers to non-adherence, medication plan and communication with other healthcare providers). ${ }^{111232}$

\section{Continuity of care}

The significance of the communication with other healthcare providers as part of a global approach to stroke survivors has been reported elsewhere. ${ }^{3132}$ The present study fully integrates the issue of continuity of care during the transition from inpatient to outpatient care by supporting patients over the year following discharge and through the transmission of a summary of interventions to the patient's GP, specialists and his/her usual community pharmacist. In this way, the study programme contributes to overcoming interprofessional boundaries by developing collaboration between hospital and community pharmacists, and between pharmacists and physicians.

\section{Randomisation}

Patient-by-patient randomisation cannot be applied to this trial as contacts between inpatients are frequent, and information sharing between two parallel groups could be expected and would constitute a major barrier to evaluating the experimental intervention. Using a period for randomisation is a logical method for this situation: each centre will be drawn to determine which period will be assigned to it for 12-18 months (intervention or standard care). A washout period of 1 month is necessary to avoid information sharing between the two groups.

\section{Measuring adherence}

The complexity of adherence to medication has hindered the development of a 'gold standard' that allows sensitive and specific evaluation and none of the published methods meets all the criteria of a reliable and reproducible measurement method. ${ }^{45}$ To obtain more reliable data, a combination of different methods is recommended. ${ }^{75}$ In this protocol we combine prescription refill as observed by the community pharmacist, which allows the distinction between prescription discontinuation and nonadherence, and a self-administered questionnaire, which are complementary and easy to implement. In particular, the selfadministered questionnaire has proven as reliable as pill counting if a quality relationship of trust exists between the caregiver and the patient. ${ }^{45}$ The advantage over other methods is to explore different dimensions of adherence. ${ }^{45}$

\begin{abstract}
Limits
This is an open-label trial in which pharmacists, physicians and patients cannot be blinded. However, we use a method to measure adherence that includes an objective evaluation so as not to influence the main outcome. Furthermore, the intervention will not be evaluated in those with cognitive impairment, aphasia or lack of autonomy which could be thought to limit the generalisation of the results. Nevertheless, it is of note that the majority of patients will be recruited from rehabilitation units that treat patients who are recovering from severe stroke, and therefore patients who potentially initially suffered from such impairments.
\end{abstract}

\section{Trial status}

Protocol version 5, effective 28 August 2019, is currently used. On 1 April 2020, 140 patients (76 in the intervention group and 64 in the standard care group) have been enrolled in the six hospital centres since 29 July 2015 . The study is still ongoing.

\section{CONCLUSION}

The ADMED-AVC study is the first trial in France to test the impact of a pharmacist-led intervention in patients with ischaemic stroke. This programme has the potential to significantly improve adherence to medication in stroke survivors, which could lead to a decrease in recurrent stroke and also other cardiovascular events, a decrease in medication iatrogenic events and a better quality of life.

\section{Author affiliations}

${ }^{1}$ Department of Pharmacy, Edouard Herriot Hospital, Hospices Civils de Lyon, Lyon, France

EA 4129 P2S Parcours Santé Systémique, Claude Bernard University Lyon 1, Lyon, France

${ }^{3}$ Physical medicine and rehabilitation department, Henry Gabrielle Hospital, Hospices Civils de Lyon, Saint-Genis-Laval, France

${ }^{4}$ INSERM U1028, CNRS UMR5292, Lyon Neuroscience Research Center, ImpAct Team, Claude Bernard University Lyon 1, Bron, France

${ }^{5}$ Public Health Center, Research and Clinical Epidemiology, Hospices Civils de Lyon, Lyon, France

${ }^{6}$ EA 7425 HESPER Health Services and Performance Research, Claude Bernard University Lyon 1, Lyon, France

${ }^{7}$ Stroke center, Pierre Wertheimer Hospital, Hospices Civils de Lyon, Bron, France

${ }^{8}$ INSERM U1060, CarMeN laboratory, Claude bernard University Lyon 1, Villeurbanne, France

${ }^{9}$ INSERM U1044, CNRS UMR 5220, CREATIS, Claude Bernard University Lyon 1, Villeurbanne, France

Acknowledgements We thank Dr Philip Robinson (Direction de la Recherche Clinique et de I'Innovation, DRCl, Hospices Civils de Lyon, Lyon, France) for help in manuscript preparation.

Contributors SK, AJD: writing the protocol, submitting the protocol to the national tender, enrolling the hospital centres, planning and setting up the study, participating in the control and interventional period. ED, SB: writing the methodology of the protocol, planning the study and performing the statistical analysis. MD: setting up the study, collecting and monitoring data. SJC, JL, LD, LM, NN, GR: co-writing and correcting the protocol, participating in the control and interventional period. CD: co-writing and correcting the protocol. All authors read and approved the final manuscript.

Funding This work was supported by the French Ministry of Health, Direction Générale de I'Offre de Soins, 14, avenue Duquesne, 75350 Paris SP 07, France (PREPS 2013 number 13-0533, supplementary files). The Hospices Civils de Lyon are the promoter and are in charge of all the administrative measures.

Competing interests None declared.

Patient consent for publication Not required.

Provenance and peer review Not commissioned; internally peer reviewed.

Data availability statement There are no data in this work.

Open access This is an open access article distributed in accordance with the Creative Commons Attribution Non Commercial (CC BY-NC 4.0) license, which permits others to distribute, remix, adapt, build upon this work non-commercially, and license their derivative works on different terms, provided the original work is properly cited, an indication of whether changes were made, and the use is noncommercial. See: http://creativecommons.org/licenses/by-nc/4.0/.

\section{ORCID iD}

Sophie Khettar http://orcid.org/0000-0002-7061-1662

\section{REFERENCES}

1 Hautes Autorité de Santé. Recommandations de bonnes pratiques. Prévention vasculaire après un infarctus cérébral ou un accident ischémique transitoire Actualisation, 2014. Available: http://www.has-sante.fr/portail/upload/docs/ application/pdf/2014-09/avc_argumentaire.pdf [Accessed 4 Jul 2018]

2 Burke JP, Sander S, Shah H, et al. Impact of persistence with antiplatelet therapy on recurrent ischemic stroke and predictors of nonpersistence among ischemic stroke survivors. Curr Med Res Opin 2010;26:1023-30.

3 Bailey JE, Wan JY, Tang J, et al. Antihypertensive medication adherence, ambulatory visits, and risk of stroke and death. J Gen Intern Med 2010;25:495-503. 
4 Lewis SJ, Robinson JG, Fox KM, et al. Underutilisation of cardiovascular medications among at-risk individuals. Int J Clin Pract 2010;64:604-10.

5 Rijkmans M, de Jong G, van den Berg JSP. Non-persistence in ischaemic stroke: risk of recurrent vascular events. Acta Neurol Scand 2018;137:288-92.

6 Lee HJ, Jang S-I, Park E-C. Effect of adherence to antihypertensive medication on stroke incidence in patients with hypertension: a population-based retrospective cohort study. BMJ Open 2017;7:e014486.

7 Sabaté E. Adherence to long-term therapies: evidence for action. Geneva: World Health Organization, 2003.

8 Glader E-L, Sjölander M, Eriksson M, et al. Persistent use of secondary preventive drugs declines rapidly during the first 2 years after stroke. Stroke 2010;41:397-401.

9 Perreault S, Yu AYX, Côté $\mathrm{R}$, et al. Adherence to antihypertensive agents after ischemic stroke and risk of cardiovascular outcomes. Neurology 2012;79:2037-43.

10 Bushnell CD, Olson DM, Zhao X, et al. Secondary preventive medication persistence and adherence 1 year after stroke. Neurology 2011;77:1182-90.

$11 \mathrm{O}^{\prime}$ Carroll RE, Chambers JA, Dennis M, et al. Improving adherence to medication in stroke survivors: a pilot randomised controlled trial. Ann Behav Med 2013;46:358-68.

12 Barker-Collo S, Krishnamurthi R, Witt E, et al. Improving adherence to secondary stroke prevention strategies through motivational interviewing: randomized controlled trial. Stroke 2015;46:3451-8.

13 Kamal AK, Shaikh Q, Pasha 0, et al. A randomized controlled behavioral intervention trial to improve medication adherence in adult stroke patients with prescription tailored Short Messaging Service (SMS)-SMS4Stroke study. BMC Neurol 2015; 15:212

14 Hornnes N, Larsen K, Boysen G. Blood pressure 1 year after stroke: the need to optimize secondary prevention. J Stroke Cerebrovasc Dis 2011;20:16-23.

15 Wan L-H, Zhang X-P, Mo M-M, et al. Effectiveness of goal-setting telephone follow-up on health behaviors of patients with ischemic stroke: a randomized controlled trial. J Stroke Cerebrovasc Dis 2016;25:2259-70.

16 Menard MM, Smith DB, Taormina C. A program to improve secondary stroke prevention: the Colorado Neurological Institute stroke preventing recurrence of thromboembolic events through coordinated treatment program. J Neurosci Nurs 2011:43:199-204.

17 McManus JA, Craig A, McAlpine C, et al. Does behaviour modification affect poststroke risk factor control? Three-year follow-up of a randomized controlled trial. Clin Rehabil 2009;23:99-105.

18 Ireland S, MacKenzie G, Gould L, et al. Nurse case management to improve risk reduction outcomes in a stroke prevention clinic. Can J Neurosci Nurs 2010;32:7-13.

19 Sit JWH, Yip VYB, Ko SKK, et al. A quasi-experimental study on a communitybased stroke prevention programme for clients with minor stroke. J Clin Nurs 2007;16:272-81.

20 Mackenzie G, Ireland S, Moore S, et al. Tailored interventions to improve hypertension management after stroke or TIA--phase II (TIMS II). Can J Neurosci Nurs 2013:35:27-34

21 Lummis HL, Sketris IS, Gubitz GJ, et al. Medication persistence rates and factors associated with persistence in patients following stroke: a cohort study. BMC Neurol 2008;8:25.

22 Hedegaard U, Kjeldsen LJ, Pottegård A, et al. Multifaceted intervention including motivational interviewing to support medication adherence after stroke/transient ischemic attack: a randomized trial. Cerebrovasc Dis Extra 2014;4:221-34.

23 McAlister FA, Majumdar SR, Padwal RS, et al. Case management for blood pressure and lipid level control after minor stroke: prevention randomized controlled trial. CMAJ 2014; 186:577-84

24 Clifford S, Barber N, Elliott R, et al. Patient-centred advice is effective in improving adherence to medicines. Pharm World Sci 2006;28:165-70.
25 Vrijens B, Belmans A, Matthys K, et al. Effect of intervention through a pharmaceutical care program on patient adherence with prescribed once-daily atorvastatin. Pharmacoepidemiol Drug Saf 2006;15:115-21.

26 Lee JK, Grace KA, Taylor AJ. Effect of a pharmacy care program on medication adherence and persistence, blood pressure, and low-density lipoprotein cholesterol: a randomized controlled trial. JAMA 2006;296:2563-71.

27 Santschi V, Chiolero A, Burnand B, et al. Impact of pharmacist care in the management of cardiovascular disease risk factors: a systematic review and meta-analysis of randomized trials. Arch Intern Med 2011;171:1441-53.

28 Machado M, Bajcar J, Guzzo GC, et al. Sensitivity of patient outcomes to pharmacist interventions. Part II: Systematic review and meta-analysis in hypertension management. Ann Pharmacother 2007;41:1770-81.

29 Machado M, Nassor N, Bajcar JM, et al. Sensitivity of patient outcomes to pharmacist interventions. Part III: Systematic review and meta-analysis in hyperlipidemia management. Ann Pharmacother 2008:42:1195-207.

30 Santschi V, Chiolero A, Colosimo AL, et al. Improving blood pressure control through pharmacist interventions: a meta-analysis of randomized controlled trials. J Am Heart Assoc 2014;3:e000718.

31 Hohmann C, Klotz JM, Radziwill R, et al. Pharmaceutical care for patients with ischemic stroke: improving the patients quality of life. Pharm World Sci 2009:31:550-8.

32 Andres J, Ruchalski C, Katz P, et al. Drug therapy management in a pharmacist-run stroke prevention clinic. Am J Health Syst Pharm 2016;73:1388-90.

33 Bergeron J, Laprise R, Mallet L. Pharmacy discharge plan for continuity in patient care. Can Pharm J 1998;131:21-3.

34 Peterson AM, Nau DP, Cramer JA, et al. A checklist for medication compliance and persistence studies using retrospective databases. Value Health 2007;10:3-12.

35 Anderson C, Laubscher S, Burns R. Validation of the Short Form 36 (SF-36) Health Survey questionnaire among stroke patients. Stroke 1996;27:1812-6.

36 Broadbent E, Petrie KJ, Main J, et al. The brief illness perception questionnaire. J Psychosom Res 2006;60:631-7.

37 Ruiz MA, Pardo A, Rejas J, et al. Development and validation of the "Treatment Satisfaction with Medicines Questionnaire" (SATMED-Q). Value Health 2008; 11:913-26

38 Manger Bouger. Programme National Nutrition Santé [Internet]. Paris: Santé publique France. Guides et documents; [about 3 screens]. Available: https://www. mangerbouger.fr/PNNS/Guides-et-documents [Accessed 4 Jul 2018].

39 Bauler S, Jacquin-Courtois S, Haesebaert J, et al. Barriers and facilitators for medication adherence in stroke patients: a qualitative study conducted in French neurological rehabilitation units. Eur Neurol 2014;72:262-70.

40 Souter C, Kinnear A, Kinnear M, et al. Optimisation of secondary prevention of stroke: a qualitative study of stroke patients' beliefs, concerns and difficulties with their medicines. Int J Pharm Pract 2014;22:424-32.

41 Kronish IM, Diefenbach MA, Edmondson DE, et al. Key barriers to medication adherence in survivors of strokes and transient ischemic attacks. J Gen Intern Med 2013;28:675-82.

42 Arif $\mathrm{H}$, Aijaz B, Islam M, et al. Drug compliance after stroke and myocardial infarction: a comparative study. Neurol India 2007:55:130-5.

43 Chambers JA, O'Carroll RE, Hamilton B, et al. Adherence to medication in stroke survivors: a qualitative comparison of low and high adherers. Br J Health Psychol 2011;16:592-609.

44 Sjölander M, Eriksson M, Glader E-L. The association between patients' beliefs about medicines and adherence to drug treatment after stroke: a cross-sectional questionnaire survey. BMJ Open 2013;3:e003551.

45 Lehmann A, Aslani P, Ahmed R, et al. Assessing medication adherence: options to consider. Int J Clin Pharm 2014;36:55-69. 